\title{
Evaluación por competencias: contextos de origen y contradicciones pedagógicas'
}

\author{
Competency Evaluation: \\ contexts of origin and educational contradictions \\ Competência Avaliação: \\ contextos de origem $\epsilon$ contradições educacionais
}

\author{
Liliana Saavedra Rey² \\ Universidad de San Buenaventura, Bogotá, Colombia \\ Sneider Saavedra Rey³ \\ Universidad Pedagógica Nacional, Bogotá, Colombia \\ Universidad de San Buenaventura, Bogotá, Colombia
}

RECIBIDO: 19 DE SEPTIEMBRE DE 2014 • APROBAdo: 20 DE OCTUBRE DE 2014

Para citar este artículo: Saavedra y Saavedra. (2014). Evaluación

por competencias: contextos de origen y contradicciones

pedagógicas. Itinerario Educativo, (64), 65-81

1 Artículo de reflexión producido en los grupos de investigación Evaluándo_nos. Pedagogía crítica, docencia y evaluación de la Universidad Pedagógica Nacional y Tendencias Actuales en Educación y Pedagogía (TAEPE) de la Universidad de San Buenaventura, Sede Bogotá.

2 Doctoranda en Humanidades. Humanismo y persona de la Universidad de San Buenaventura. Magíster en Educación en la línea de Evaluación educativa, Especialista en Gerencia social de la Educación y Licenciada en Preescolar de la Universidad Pedagógica Nacional. Actualmente es Coordinadora de Investigaciones de la Facultad de Educación de la Universidad San Buenaventura y directora del grupo de investigación Tendencias Actuales en Educación y Pedagogía (TAEPE). Bogotá, Colombia. Correo electrónico: lsaavedra@usbbog.edu.co

3 Magíster en Educación en la línea de Evaluación educativa y Licenciado en Humanidades, Español y lenguas extranjeras de la Universidad Pedagógica Nacional. Actualmente es Coordinador del área de Español y literatura del Gimnasio Los Portales y profesor de la Facultad de Educación de la Universidad Pedagógica Nacional y Universidad de San Buenaventura. Profesor investigador de los grupos Evaluándo_nos. Pedagogía crítica, docencia y evaluación (UPN) y Tendencias Actuales en Educación y Pedagogía (TAEPE) (USB). Bogotá, Colombia. Correo electrónico: sneider201@hotmail.com. 
Resumen. Este artículo presenta los contextos de origen del concepto competencia con el propósito de establecer una versión ampliada que permita reconocer su génesis en la lingüística, su desarrollo en la psicología cognitiva y su inserción al discurso neoliberal en la planificación de los sistemas escolares, desde el Estado hasta las empresas privadas. De esta manera, se propone un ámbito pedagógico que evidencia las contradicciones y posibilidades de este proyecto educativo, en el reconocimiento de la importancia de la evaluación para consolidar tales propuestas. Así, se plantea reemplazar la calificación por la evaluación, las competencias por la actuación, las fronteras disciplinares por la transdisciplinariedad, los tiempos curriculares por el de la indagación, el mundo neoliberal por el de la vida.

Palabras clave. Competencia, actuación, evaluación, políticas educativas, currículo, neoliberalismo (Tesauro Unesco).

Abstract. This article presents the inception contexts of the concept competence in order to establish a extended version that recognizes its genesis in linguistics, its development in cognitive psychology and its incorporation to the neoliberal discourse in the school system planning, from the State to the private enterprises. For this reason, the article proposes a pedagogic sphere that shows contradictions and posibilities of this educative project, from the importance of evaluation to consolidate this kind of proposals. That way, it is proposed an alternative to replace evaluation for assessment, competence for performance, disciplines boundaries for transdisciplinarity, curriculum time for the one dedicated to inquiry and the neoliberal world for the life-world.

Keywords. Competence, performance, evaluation, educative policy, curriculum, neoliberalism (Unesco Thesaurus).

Resumo. Este artigo apresenta os contextos de origem do conceito de competência, a fim de estabelecer uma versão expandida que reconhece a sua génese em lingüística, o seu desenvolvimento em psicologia cognitiva e sua inserção ao discurso neoliberal nos sistemas de ensino de planejamento, por parte do Estado para empresas privadas. Assim, um campo educacional que mostra as contradições e possibilidades deste projeto educativo, em reconhecimento da importância da avaliação para consolidar essas propostas é proposto. Assim, propõe-se a substituir a avaliação de rating, as habilidades para agir, as fronteiras disciplinares pela transdisciplinaridade, o tempo curricular para o inquérito, o mundo neoliberal para a vida.

Palavras-chave. Competência, desempenho, avaliação, política educacional, currículo, o neoliberalismo (Unesco Thesaurus). 


\section{Introducción}

La multiplicidad de definiciones y mixtura de orígenes del concepto competencia no permite un abordaje unívoco, definitivo o ingenuo pues atraviesa ámbitos tan contradictorios como la psicología cognitiva, la psicometría, la gestión de recursos humanos, los estudios del lenguaje, la pedagogía y las políticas educativas. Una "palabra plástica", según Del Rey (2012), usualmente al servicio de diversos intereses, que reina en medio de la confusión pedagógica, manteniéndose en el primer orden de los propósitos de los sistemas de educación en el mundo.

Si bien este contexto precisa una actuación del profesorado para formar ciudadanos competentes, flexibles en su pensamiento, adaptables a las transformaciones sociales, con conocimientos útiles y aplicables al mundo real; el sistema escolar promovido por las mismas políticas carece de las condiciones para desarrollar tales procesos: fronteras disciplinares infranqueables, tiempos segmentados por área, maestros con sobrecarga laboral, dificultades para proveer diversos contextos de actuación según la variedad de estilos y ritmos de aprendizaje y, sobre todo, incoherencias de un sistema evaluativo que privilegia las pruebas censales o sus sucedáneos en el aula de clase.

Precisamente, esta evaluación por competencias ha resultado contradictoria porque se ha articulado a un sistema anterior para medir rendimientos, cuya estrategia principal es la estandarización, antípoda de los saber-hacer en contexto, siempre individuales y situados. Más aún, debido a que la evaluación plantea "lo deseable" de la educación (Bretel y Crespo, 2006), estas imprecisiones conllevan relativismos didácticos y curriculares, desde el inocuo cambio de redacción de los otrora objetivos o logros, ahora bajo el título de competencias, desempeños o actuaciones; hasta la indefinición de tales conceptos en los currículos oficiales, pasando indefectiblemente por prácticas que no configuran escenarios pertinentes para las competencias, no por reacción política del profesorado a sus demandas, sino por simple omisión, confusión o ignorancia sobre su abordaje.

En esencia, la propuesta de formar en conocimientos transferibles al mundo real no es perjudicial. Es más, a la luz de la transmisión enciclopédica de saberes de gran auge en otros momentos históricos, presenta bondades y respuestas coherentes a los desafíos de la sociedad actual.

ITINERARIO EDUCATIVO • ISSN OIZI-2753 • AÑO XXVIII, N. ${ }^{\circ} 64$ • JUUIO - DICIEMBRE DE 20I4 • P. 65-8I 
Sin embargo, la inserción de las competencias a la educación - su "naturalización" - tanto por la manera en que se ha desarrollado como por la ambigüedad de sus orígenes, ha generado un sistema incoherente entre propósitos, medios y formas de evaluar. Por esta razón, este artículo plantea la génesis de las competencias y sus múltiples contextos de origen, con el fin de establecer una definición ampliada de este concepto. A partir de allí, establece sus contradicciones pedagógicas, especialmente en el campo evaluativo, vislumbrando las transformaciones requeridas si en realidad se pretende la formación de personas competentes para la vida en complejidad, y no remedos de activismo o de gestión de recursos humanos como innovación de la escuela.

\section{Una versión ampliada de la definición de competencias}

La indeterminación de un concepto justifica su abordaje y uso indiscriminados. Por esta razón, simplificar la competencia a un término indefinible significa dejarlo a la deriva de las intencionalidades de cualquier ámbito social, con las implicaciones discursivas que esto acarrea, del mismo modo que abordar solo una de sus acepciones es tergiversar o limitar sus posibilidades. Como afirma Díaz Barriga (2006), se ha generalizado la negativa pedagógica de abordar la conceptualización de las competencias, lo cual ha acarreado su aplicación superficial u orientaciones insuficientes en el ámbito educativo, muchas veces justificadas simplemente por su carácter innovador. Aunque la noción de competencia se ha configurado desde ámbitos diversos, es posible establecer una caracterización que recoja tales acepciones y contextos de origen - una versión ampliada - en cuya constitución divergen, no tanto las conceptualizaciones, sino las intencionalidades y usos a los que se ha visto abocado el término, desde definir un procesamiento mental en una investigación lingüística hasta abanderar un proyecto político para adecuar la educación a las demandas laborales.

Tan solo Moreno (2009) en un rastreo panorámico del concepto encuentra más de trece clasificaciones: por ejemplo, mientras Mansfield (2009) las relaciona con resultados, tareas y rasgos personales, Weinert (1999) enlista nueve formas diferentes de competencia (habilidades cognitivas especializadas, modelo de actuación competente, autoconcepto objetivo y subjetivo, metacompetencias, entre otras). Otro tanto afirma Bustamante (2003), quien evidencia las acepciones del concepto de acuerdo con su 
relación con los estudios del lenguaje y la educación: competencias ideológica, enciclopédica, discursiva, semántica, pragmática, hermenéutica, poética; y epistémica, metodológica e investigativa, respectivamente. Díaz Barriga (2006), por su parte, realiza otra clasificación: las competencias genéricas, constituidas por las académicas y las que son para la vida; las competencias disciplinares y transversales según un criterio curricular; las complejas, derivadas y subcompetencias de la formación profesional; y finalmente, en cuanto al desempeño profesional, enuncia competencias básicas, iniciales y avanzadas.

Muchos otros, Barnett (2001), Perrenoud (2004), Van der Klink et. al. (2007) y Maldonado (2011), han abordado las competencias con idénticos resultados: profusión, ambigüedad, vastas enumeraciones. No operan claridades al respecto "ni una clasificación completa, racional y funcional que oriente los procesos de diseño curricular y los sistemas de enseñanza" (Díaz Barriga, 2006, p. 33) Es más, un catálogo tal no resulta conveniente para el profesorado que debe formar en competencias sin saber, en definitiva, a lo que refieren. Por esta razón, a continuación se plantearán los contextos de origen del concepto, su definición e intencionalidades, de manera que los maestros reconozcan, no su unidad inexistente, sino las múltiples implicaciones de este tipo de educación.

\section{La génesis teórica del concepto: competencias lingüística y comunicativa}

En su obra Aspectos de la teoría de la sintaxis, Chomsky (1975) define la competencia lingüística como la capacidad innata de todo ser humano para adquirir el conocimiento de una lengua. Este dominio (competence) permite una actuación (performance) en tanto aplicación de dicho conocimiento a una situación determinada ${ }^{4}$. Si bien este autor acuña el término para el campo de la lingüística, es importante reconocer, con Bustamante (2010), que no es un concepto nuevo sino que retoma los planteamientos de Humboldt para precisar que la facultad del lenguaje

4 De acuerdo con el interés investigativo de Chomsky, esta situación es "idealizada", es decir, constituida por "hablantes-oyentes ideales", en una "comunidad lingüística homogénea", aunque la realidad precisamente se distinga por la imposibilidad de que tales condiciones existan. Aunque sus detractores consideren este aspecto como un descuido imperdonable, el lingüista lo plantea de esta manera porque su propósito es construir una teoría mentalista al respecto, no describir las situaciones comunicativas de hecho. 
supone un proceso generativo gracias a la competencia subyacente de todos sus usuarios. Asimismo, debido al interés chomskiano de retomar esta lingüística mentalista basada en una gramática (competencia) en cuyo dominio y uso de reglas universales y finitas se puedan generar infinito número de oraciones, el autor privilegió el estudio de la competencia (conocimiento de la lengua) sobre la actuación (el uso o aplicación de dicho conocimiento), la cual simplemente evidenciaría externamente lo que sucede en la mente.

Esta segunda noción constitutiva es enfatizada por Hymes (1996) para desarrollar la noción de competencia comunicativa pues, evidentemente, el carácter mentalista de la gramática generativa no logra explicar las contingencias de la comunicación. Así, la competencia comunicativa retoma la competencia lingüística como conocimiento de la lengua pero la contextualiza, enfatizando en las eventualidades que se pueden presentar en la actuación y la manera en que, en definitiva, lo que hacen los usuarios de la lengua es "adecuarse" a la situación en la que se encuentran. Por ello, se define como "el conjunto de procesos y conocimientos de diverso tipo - lingüísticos, sociolingüísticos, estratégicos y discursivos - que el hablante/oyente/escritor/lector deberá poner en juego para producir o comprender discursos adecuados a la situación y al contexto de comunicación y al grado de formalización requerida" (Hymes, 1996, p. 15).

Esta acepción de competencia consolida la articulación entre conocimiento y acción contextualizada que se ha insertado a la educación como "saber-hacer en contexto" bajo el supuesto de que la competencia comunicativa es aplicable a cualquier saber. Esta actuación evidencia la complejidad del conocimiento de las personas porque implica adquirirlo, utilizarlo y adecuarlo según las exigencias de una situación determinada, a la manera de la resolución de problemas: no basta con saber un algoritmo si no se comprende la situación en que puedo aplicarlo para resolver una situación de la vida real.

\section{La psicología cognitiva y la inserción de las competencias a la educación}

El paso de las competencias a la educación difícilmente se hubiera logrado sin la relevancia que adquirió en su momento el concepto para la psicología, disciplina que, desde el siglo pasado, se erigió como referente 
importante para las propuestas pedagógicas. En este sentido, Torrado (1999) parte de la formulación chomskiana para documentar la manera en que este modelo de funcionamiento lingüístico se extendió a todo el sistema cognitivo, precisamente desde la célebre revolución cognitiva de los años sesenta. En efecto, el concepto de competencia replanteó la teoría piagetiana propiciando el desarrollo de la psicología cognitiva de corte computacional, al proponer que no todas las actividades cognitivas estaban subordinadas a una lógica mental general, sino que poseían una lógica de funcionamiento específica o modular para cada dominio de la actividad mental (como exponía Chomsky al referirse al procesamiento lingüístico).

De ahí se deriva el uso extendido de la competencia cognitiva como aquel dispositivo idealizado que representa la capacidad mental para desarrollar y ampliar conocimientos, junto a las habilidades, actitudes y destrezas que constituyen dicho proceso de aprendizaje. De acuerdo con Gallego (2000), este concepto establece la relación entre la inteligencia como aptitud formable y las actitudes consecuentes que potencian las destrezas y habilidades de cada individuo gracias a sus interacciones con un colectivo.

De manera paralela a la propuesta de Hymes (1996) y su énfasis en la actuación, en el campo de la psicología Vigotsky (2010) promovió su perspectiva cultural en la cual todas las funciones psicológicas superiores son inicialmente sociales y luego se internalizan, por lo cual predomina la mediación cultural significativa para lograr el aprendizaje. Con esta fundamentación, también se critica la visión mentalista, en este caso de Piaget, pues los dispositivos cognitivos dependen de las interacciones sociales que los configuran y a los que deben responder en el uso de sus conocimientos derivados. Nuevamente, se impone la idea de contexto, que seguirá desarrollando Bruner (1986) cuando explota la relación entre la competencia lingüística gramatical y la mediación de los adultos para que los niños construyan formatos de adquisición de lenguaje. Es decir, en el proceso de aprendizaje se reconoce tanto el dispositivo innato que lo hace posible como la mediación social que lo apoya.

La competencia como una nueva conceptualización es diferente por ello a los conceptos de aptitud o de capacidad mental manejados tradicionalmente, pues no se trata de una capacidad potencial con la cual se nace, sino de un saber hacer, que para desplegarse debe ser 
aprendido de la cultura y con la ayuda del otro, así se posean múltiples dispositivos de reglas del funcionamiento cognitivo, que son condición básica; la competencia no es una actitud, ni una capacidad, que se tiene y que por tanto se puede manifestar siempre y en todo lugar, idéntica, y tampoco es la misma para todos y en todo (Torrado, 1999, p. 29).

La pertinencia para explicar el procesamiento de información y el proceso de aprendizaje derivado hizo de la psicología cognitiva y la psicología cultural referentes fundamentales para la educación en las últimas décadas. Quizá la mayor muestra de este apoyo disciplinar sea el constructivismo, cuyo planteamiento puede leerse a la luz de la dicotomía competencia-actuación, de donde se desprende la idea de la ampliación de redes conceptuales y la construcción de conocimientos a partir de experiencias significativas y saberes previos. Justamente, el impacto de la psicología cognitiva y la psicología cultural en la educación propició el engranaje necesario para el paso expedito de las competencias al campo educativo.

\section{Las competencias como proyecto político y económico}

Tal como afirma Maldonado (2011), no es el discurso teórico sino el político el que ha prevalecido desde mediados del siglo pasado para desarrollar el tema de las competencias. Tampoco es coincidencia que durante este período hayan surgido organismos internacionales como la Organización Internacional del Trabajo (OIT), el Banco Mundial (BM), la Organización Mundial del Comercio (OMC), el Centro Europeo para la Formación Profesional (Cedefop), la Organización Económica de Cooperación para el Desarrollo (OCDE), el Proyecto Tuning, el Consejo de Normalización y Certificación de Competencia Laboral (Conocer), entre otros interesados en la formación de la flexibilidad y la adaptabilidad de los sujetos para afrontar las condiciones laborales y sociales cambiantes del orden económico neoliberal. En efecto, este fenómeno trasciende los límites locales en tanto política transnacional y ha encontrado en el discurso de las competencias el mejor asidero para involucrar los intereses económicos a la educación desde la aparición de la Competency-Based Education and Training estadounidense en los años sesenta, así como sus actualizaciones y sucedáneos alrededor del mundo.

De acuerdo con Del Rey (2012), quien ha indagado esta tendencia mundializada en diversos países de la Comunidad Europea y América 
latina, el cruce de tres procesos ha posibilitado la aparición de las competencias como parte de este proyecto político y económico: la variante psicométrica de la psicología cognitiva y su medición de "aptitudes", la planificación de los sistemas educativos a nivel mundial basada en la economía de la educación y la gestión de recursos humanos de acuerdo con los perfiles laborales planteados por las empresas.

El primer proceso se relaciona con la creación de la Association for the Evaluation of Educational Achievement (IEA) en 1952 - primer organismo internacional de evaluación de resultados escolares - y la taxonomía de objetivos educativos propuesta por Benjamin Bloom. De esta manera se plantea por primera vez "una correspondencia entre los objetivos en cuestión y las aptitudes cognitivas. Y como estas actitudes se consideran universales por haber sido promovidas por la psicología cognitiva, he aquí que esos objetivos se volverían, en virtud de ese fundamento científico, estándares para la evaluación" (Del Rey, 2012: 68). Otros desarrollos históricos contribuyeron a esta condensación de una práctica calificativa a gran escala: la perspectiva técnica de la evaluación basada en la administración científica del trabajo de Frederick Taylor que permitió asumir la medición de resultados escolares a la manera de las producciones industriales, con criterios de eficiencia y eficacia; la propuesta curricular por objetivos de Ralph Tyler (1986) que, como vicaria de la anterior, determinó los objetivos de la enseñanza en términos medibles y cuantificables; y los avances en materia de test de inteligencia de parte de psicólogos como Binet y Simon, a quienes se confió la medición de las aptitudes cognitivas de los estudiantes estadounidenses.

En el contexto posterior a la Segunda Guerra Mundial, la explosión demográfica y los sistemas escolares de masas derivados conllevaron a planificar la educación de acuerdo con los recursos a disposición de los gobiernos. Este segundo proceso, desarrollado bajo la influencia de organismos internacionales como la Unesco y teorías de la economía de la educación en boga como la de Shultz (1983), reconoció la importancia de invertir en educación en tanto capital humano para generar rentabilidad y contribuir al crecimiento económico de los Estados. Como las competencias implican no solo un conocimiento sino su uso en contexto y la posibilidad de refinarlo y reflexionarlo, construyéndolo socialmente y consolidándolo como aptitud para próximas actuaciones, este proyecto político y económico lo acuñó en clave de su flexibilidad y adaptabilidad a las situaciones sociales cambiantes, así como a la proyección continua 
de aprendizaje, que en este nuevo orden, implica la cualificación constante y la inversión en el capital humano propio.

Finalmente, la derivación de este fenómeno implicó también a las empresas como directas responsables de este proyecto económico. En este marco de desinversión del Estado y de cesión de responsabilidades a los individuos, las organizaciones empresariales comenzaron a subsanar los vacíos o potenciar los aciertos del sistema educativo de acuerdo con los perfiles laborales requeridos. Con este propósito se tecnificó la selección, el reclutamiento y la capacitación continua de los empleados a través de programas e instituciones especializadas en recursos humanos. En su seno, las competencias se convierten en un concepto maleable que permite, al mismo tiempo, reconocer el valor de aspectos inmateriales en los trabajadores, la acumulación de experiencia relativa a su saber-hacer, su nivel de adaptabilidad y manejo de sí en situaciones de incertidumbre, el establecimiento de relaciones sociales, su toma de decisiones efectivas, entre infinidad de aspectos cognitivos, actitudinales y procedimentales, según el menú de intereses de la empresa en cuestión.

A partir del momento en que se impuso la idea de que una empresa competitiva es una empresa que sabe invertir en su capital cognitivo (la suma de las competencias de sus empleados), la idea de que la empresa podría ganar formando a sus propios empleados se ha impuesto a su vez; y, de allí, la idea de que la empresa sería por sí misma formadora en la medida en que el trabajo pone en marcha competencias bien específicas. El modelo que actualmente vende la empresa de sí misma es el de una "organización aprendiente": el empleado trabaja, al trabajar se forma $y$, al formarse, valora a la empresa que lo valora, lo que la valoriza, y así sucesivamente (Del Rey, 2012, p. 87).

Debido a estos tres procesos, la misma autora define esta inserción de las competencias a la educación como una estrategia "neoliberal tendiente a ubicar, más que la educación al servicio del hombre, el pequeño hombre a ser educado al servicio de las necesidades de la economía" (Del Rey, 2012, p. 25). Esto propone, sin duda, un cuestionamiento más profundo sobre la caracterización y el valor de lo humano para la sociedad actual, que sitúa como realización personal el éxito laboral derivado de invertir en competencias, en capital humano. Este proyecto político y económico ha vertido los avances teóricos y psicológicos presentados en aspectos medibles y cuantificables de la actuación para el ámbito laboral, como si 
todo conocimiento tuviera que derivar en réditos, como si la formación humana se circunscribiera al crecimiento económico.

Así pues, esta versión ampliada del concepto competencia propone, para los maestros, la toma de conciencia en el momento de realizar propuestas educativas encaminadas en este sentido, pues en su desarrollo y aplicación, quiérase o no, se reproducen estos presupuestos e intencionalidades: un sesgo psicológico dentro de la formación, enfocado exclusivamente en el aprendizaje; la manera en que este aprendizaje se logra y justifica con su uso en contextos reales y específicos; la concepción de que esta aptitud para aprender se construye y reconstruye progresivamente, mediada por la actuación, lo cual genera y fortalece ciertas habilidades y destrezas; y finalmente, el uso de las concepciones anteriores para adelantar un proyecto neoliberal que restringe la educación al ámbito laboral, desde el Estado hasta la empresa privada.

\section{Contradicciones pedagógicas de la evaluación por competencias}

Esta versión ampliada de las competencias evidencia que sus contextos de origen y expansión no refieren a la pedagogía, más allá de sus acercamientos cognitivos y las discusiones suscitadas por su inserción a la educación. De esta manera, se excluye el saber del maestro, restándole autonomía a su profesión por intereses de otros sectores sociales (Hernández y Sánchez, 1999; Imbernón, 1994; Marcelo, 2001), en los que prima "la construcción de una aptitud general para actuar eficazmente" (Del Rey, 2012, p. 101). Así, el profesorado desvirtúa su conocimiento en atención a las prescripciones de los paquetes políticos sobre lo que los ámbitos social, económico y laboral demandan. Esto ha invertido el orden de la tríada educación-conocimiento-sociedad para hacer que este último componente sea el que configure lo que es el conocimiento y lo que debe enseñar la escuela (Barnett, 2001). Ahora bien, si tan diversos entramados ideológicos han utilizado las competencias y tal uso estratégico hace inevitable su permanencia en la educación, ¿̇por qué no reconstruirlas en el discurso pedagógico para la formación humana?

En este sentido, el propósito de usar los conocimientos en la vida real implica asumir al menos los siguientes cuestionamientos pedagógicos: 
¿Qué se entiende por conocimiento y vida real? ¿Por qué pretender que todos los conocimientos sean útiles? ¿Para quién se consagra tal utilidad? ¿Cómo se aborda la noción de contexto real en la educación y la evaluación por competencias? ¿Cómo evaluar estas competencias si solo se accede a la actuación de los educandos? ¿Las condiciones curriculares del sistema escolar actual posibilitan un proyecto educativo en este sentido?

Una educación basada en competencias es incoherente si no se basa de manera auténtica en las nociones de conocimiento, proceso y contexto. Como se ha visto, una competencia no es solo acción, sino una actuación contextualizada, "resultado de la movilización, integración y adecuación de conocimientos, habilidades y actitudes" (Villardón, 2006, p. 60). Es decir, la base de la competencia es el conocimiento, por lo cual pierden sentido las propuestas didácticas y evaluativas que lo simplifican en un desempeño desprovisto de saberes. De la misma manera, el conocimiento no es entidad absoluta sino que se construye y enriquece socialmente, en la interacción con otros, en múltiples actuaciones, como proceso, por lo cual se desvirtúan los resultados definitivos que proyecta la "racionalidad técnica de la evaluación" (Álvarez Méndez, 2001; Santos Guerra, 1998) y sus estándares derivados de la variante psicotécnica anteriormente expuesta. En tercer lugar, el contexto real es el mayor desafío de un sistema educativo que, por su misma naturaleza, presenta dificultades en cuanto a la escolarización de prácticas sociales dinámicas, complejas e implícitas, que debe mostrar como secuenciales, fragmentadas y públicas con el propósito de hacerlas enseñables (Lerner, 2001). Abrir las puertas del aula al mundo real en su complejidad es una asignatura pendiente de la mayoría de centros escolares en el mundo.

Paradójicamente, tales contradicciones constituyen una posibilidad valiosa para transformar estos anquilosamientos del sistema educativo, abriendo los currículos a abordajes transdisciplinares que realmente desarrollen propuestas didácticas como la enseñanza para la comprensión, el aprendizaje cooperativo, los estudios de caso, los proyectos transversales, la resolución de problemas, el aprendizaje significativo o el pensamiento crítico. Igualmente, posibilita aperturas curriculares para pensar en complejidad los saberes, comprender su sentido y devolverle a la escuela su trascendencia social, como institución de formación, superando su dimensión de capacitación laboral. En efecto, el estado actual del concepto de competencias se enfoca en el para qué de la educación, 
con un sesgo utilitarista exacerbado, propio del "discurso de la calidad educativa" (Santos Guerra, 2003), pero su amplitud permite y justifica establecer los porqué propios de la pedagogía, la construcción de sentido de lo que se hace en la escuela y sin lo cual no se logra aprendizaje (Vigotsky, 2010) ni formación (Garcés y Runge, 2011).

Como se ha planteado en otro lugar, la evaluación se constituye en ese campo de tensiones que evidencia lo deseable de las propuestas educativas, su deber ser en la articulación de una presión social (la certificación) y una acción formativa (Saavedra y Saavedra, 2013). Allí mismo se ha diferenciado la racionalidad técnica (calificación de rendimientos a través de puntuaciones con pretensión objetiva) de la racionalidad práctica y crítica (evaluación procesual para la comprensión y mejora del aprendizaje) (Álvarez Méndez, 2001; Santos Guerra, 1998). En el caso de las competencias, la desarticulación entre teoría y técnica ha hecho que prevalezca la aplicación de esta última en cuanto medición sin fundamentos conceptuales para un desarrollo coherente (Díaz Barriga, 2006). En dicho terreno, el proyecto de educar por competencias denota su uso estratégico en el discurrir político, económico y académico, al tiempo que devela sus inconsistencias curriculares y evaluativas pues no se han logrado las condiciones escolares para su desarrollo auténtico. Tal "desarrollo", en efecto, refiere a un proceso imposible de calificar en los sistemas estandarizados actuales porque no se puede medir una competencia que se forma internamente; en cuyo caso lo más cercano es el abordaje de las actuaciones, desde las cuales se podrían inferir aspectos de las competencias (Bustamante, 2010; Villardón, 2006). Además, tales competencias son internas, personales y subjetivas, por lo cual no podrían articularse a estándares constituidos externamente a cada persona, y menos desde un abordaje universal, sin atender a los diferentes procesos de formación y ritmos de aprendizaje.

Por esta razón, asumir la evaluación por competencias implica, por lo menos, transformar los sistemas escolares en cuanto a los tiempos, espacios y saberes segmentados; la construcción de contextos auténticos y enriquecidos para el aprendizaje; y la constitución de otras alternativas para evaluar, pues las existentes contradicen la idea de un saber-hacer en contexto, el carácter subjetivo de la competencia y la noción de transferencia a la vida real. Asimismo, implica deconstruir ese sentido de "vida real" referido a la capacitación para el mundo laboral y asumir una educación para el "mundo de la vida" (Barnett, 2001), en el cual 
no todos los conocimientos, y menos aún la formación, son medibles, cuantificables ni al servicio de algún campo particular. También existen bienes simbólicos inconmensurables (Díaz Barriga, 2000) y todo tipo de conocimientos, aptitudes y habilidades que no se desarrollan para ser "usados" en la vida, pero que constituyen el ser profundo de la realidad humana. Esos que, poéticamente, Savater (2002) distancia de los que usan las personas para "lograr vivir" (interés técnico) y que considera fundamentales para "querer vivir".

En conclusión, la educación actual no consolida una evaluación por competencias porque tal concepción, desde su misma enunciación, es contradictoria, más aún con las condiciones reales de los sistemas educativos: no es evaluación (racionalidad práctica y crítica) sino calificación (racionalidad técnica), y no es sobre competencias (imposibles de evidenciar) sino sobre sus actuaciones derivadas. Además, la educación por competencias está sesgada al discurso político que pondera las más beneficiosas en términos económicos y relega otras fundamentales para la formación humana. En este contexto, si se quiere adelantar el proyecto educativo por competencias es necesario efectuar múltiples cambios didácticos, curriculares y evaluativos desde el campo pedagógico, aprovechando la amplitud del concepto para reformar la escuela y potenciar la construcción de sentido de los estudiantes en las prácticas cotidianas que allí se realizan. Esto solo se logra con la apertura de disciplinas y tiempos escolares a la construcción transdisciplinar en contextos reales y diversificados del mundo de la vida, de acuerdo con las posibilidades y estilos de aprendizaje de los estudiantes: instituciones indagadoras, que aborden problemáticas de su realidad social y científica para potenciar sus construcciones colectivas.

Una educación por competencias riñe con la perspectiva técnica de la evaluación, negando cualquier posibilidad de estandarización, la cual no solo limita por enunciar mínimos de aprendizaje, sino que resulta incoherente porque supone resultados (no procesos), medibles (con respecto a una competencia inconmensurable), estandarizados u objetivos (no subjetivos), externos (no internos) en contextos simulados (no reales). Si el propósito es educar por competencias, su evaluación debe ser coherente, basada en la valoración cualitativa e individual de las actuaciones de los educandos en contextos de la vida real a los cuales la escuela debe abrir la puerta. 


\section{Referencias}

Álvarez Méndez, J. M. (2005). Evaluar para comprender, examinar para excluir. Madrid: Morata.

Barnett, R. (2001). Los límites de la competencia. El conocimiento, la educación superior y la sociedad. Barcelona: Gedisa.

Bretel, L. y Crespo, E. (2006). La evaluación como medio para asegurar los aprendizajes. http://breteleando.blogspot.com. Publicado en la red en noviembre de 2006. Consultado el 15 de septiembre de 2010.

Bustamante, G. (2003). El concepto de competencia III. Un caso de contextualización. Bogotá: Sociedad Colombiana de Pedagogía.

Bustamante, G. (2010). Competencia lingüística y educación. En Folios, 31, pp. 81-90.

Bruner, J. (1986). El habla del niño. Aprendiendo a usar el lenguaje. Barcelona: Paidós.

Chomsky, N. (1975) Aspectos de la teoría de la sintaxis. Madrid: Aguilar.

Del Rey, A. (2012). Las competencias en la escuela. Una visión crítica sobre el rendimiento escolar. Buenos Aires: Paidós.

Díaz Barriga, A. (2006). El enfoque de competencias en la educación. ¿Una alternativa o un disfraz de cambio? En Perfiles educativos. Vol. XXVIII (111), pp. 7-36.

Díaz Barriga, A. (2000). Evaluar lo académico. Organismos internacionales, nuevas reglas y desafíos. México: Fondo de cultura económica.

Gallego, R. (2000). El problema de las competencias cognoscitivas, una discusión necesaria. Bogotá: Universidad Pedagógica Nacional.

Garcés, J. y Runge, A. (2011). Educabilidad, formación y antropología pedagógica: repensar la educabilidad a la luz de la tradición pedagógica alemana. En Revista Científica Guillermo de Ockham. 9 (2). pp. 13-25.

Hernández, F. y Sánchez, J. (1999). Para enseñar no basta con saber la asignatura. Barcelona: Paidós.

Hymes, D. (1996). Acerca de la competencia comunicativa. En Forma y Función. 9. pp. 13-37.

ITINERARIO EDUCATIVO • ISSN OIDI-2753 • AÑO XXVIII, N. 64 • JULIO - DICIEMBRE DE 20I4 • P. 65-8I 
Imbernón, F. (1994). La formación del profesorado. Barcelona: Paidós

Lerner, D. (2001). Leer y escribir en la escuela: lo real, lo posible, lo necesario. México: Fondo de Cultura Económica.

Mansfield, B. (2004), «Competence in transition», Journal of European Industrial Training, 28 (2/3/4), pp. 296-309.

Maldonado, M. A. (2011). Currículo con enfoque de competencias. Bogotá: ECOE.

Marcelo, C. (2001). (Ed.) La función docente. Madrid: Síntesis.

Moreno, T. (2009). Competencias en educación superior: un alto en el camino para revisar la ruta de viaje. En Perfiles educativos, Vol. XXXI (124). pp. 69-92.

Perrenoud, P. (2004). Díez nuevas competencias para enseñar. Barcelona: Grao.

Saavedra L. y Saavedra S. (2013) De lo deseable a lo realizable: didáctica, currículo y evaluación. En: Niño Zafra, L. (Comp.) (2013) Currículo y evaluación críticos: pedagogía para la autonomía y la democracia. Bogotá: Universidad Pedagógica Nacional.

Santos Guerra, M.A. (1998). Evaluar es comprender. Buenos Aires: Magisterio del Río de la Plata.

Santos Guerra, M.A. (coord.) (2003). Trampas en educación. El discurso sobre la calidad. Madrid: Muralla.

Savater, F. (2002). Instrucciones para olvidar al Quijote. Barcelona: Taurus.

Shultz, T. (1983). La inversión en capital humano. En Educación y Sociedad. Vol. 8 (6).

Torrado, M.C. (1999). Educar para el desarrollo de las competencias. Bogotá: ICFES.

Tyler, R. (1986). Principios básicos del currículo. Buenos Aires: Troquel.

Van Der Klink, M. et. al. (2007). Competencias y formación profesional superior: presente y futuro. En Revista europea de formación profesional. pp. 74-91.

Vigotsky, L. (2010). Pensamiento y lenguaje. Barcelona: Paidós. 
Villardón, L. (2006). Evaluación del aprendizaje para promover el desarrollo por competencias. En Educatio Siglo XXI, 24. pp. 57-76.

Weinert, F.E. (2001), «Concept of competence: A conceptual clarification», en D.S. Rychen y L.H. Salganik (eds.), en Definition and selection key competencies, Gottingen, Alemania, Hogrefe \& Huber, pp. 45-65. 